\title{
Trends and Costs of External Electrical Bone Stimulators and Grafting Materials in Anterior Lumbar Interbody Fusion
}

\author{
Anthony D’Oro ${ }^{1}$, Zorica Buser ${ }^{1}$, Darrel Scott Brodke ${ }^{2}$, Jong-Beom Park ${ }^{3}$, Sangwook Tim Yoon ${ }^{4}$, \\ Jim Aimen Youssef ${ }^{5}$, Hans-Joerg Meisel ${ }^{6}$, Kristen Emmanuel Radcliff ${ }^{7}$, Patrick Hsieh ${ }^{8}$, Jeffrey Chun Wang ${ }^{1}$ \\ ${ }^{I}$ Department of Orthopaedic Surgery, Keck School of Medicine, University of Southern California, Los Angeles, CA, USA \\ ${ }^{2}$ Department of Orthopedics, University of Utah School of Medicine, Salt Lake City, UT, USA \\ ${ }^{3}$ Department of Orthopaedic Surgery, Uijongbu St. Mary's Hospital, College of Medicine, The Catholic University of Korea, Uijongbu, Korea \\ ${ }^{4}$ Department of Orthopedics, Emory Spine Center, Atlanta, GA, USA \\ ${ }^{5}$ Spine Colorado, Durango, CO, USA \\ ${ }^{6}$ Department of Neurosurgery, Bergmannstrost Hospital, Halle, Germany \\ ${ }^{7}$ Rothman Institute, Thomas Jefferson University, Egg Harbor Township, NJ, USA \\ ${ }^{8}$ Department of Neurological Surgery, Keck School of Medicine, University of Southern California, Los Angeles, CA, USA
}

\begin{abstract}
Study Design: Retrospective review.
Purpose: To identify the trends in stimulator use, pair those trends with various grafting materials, and determine the influence of stimulators on the risk of revision surgery.

Overview of Literature: A large number of studies has reported beneficial effects of electromagnetic energy in healing long bone fractures. However, there are few clinical studies regarding the use of electrical stimulators in spinal fusion.

Methods: We used insurance billing codes to identify patients with lumbar disc degeneration who underwent anterior lumbar interbody fusion (ALIF). Comparisons between patients who did and did not receive electrical stimulators following surgery were performed using logistic regression analysis, chi-square test, and odds ratio (OR) analysis.

Results: Approximately $19 \%$ of the patients $(495 / 2,613)$ received external stimulators following ALIF surgery. There was a slight increase in stimulator use from 2008 to 2014 (multi-level $R^{2}=0.08$, single-level $R^{2}=0.05$ ). Patients who underwent multi-level procedures were more likely to receive stimulators than patients who underwent single-level procedures $(p<0.05 ; 0 R, 3.72 ; 95 \%$ confidence interval, 3.02-4.57). Grafting options associated with most frequent stimulator use were bone marrow aspirates (BMA) plus autograft or allograft for single-level and allograft alone for multi-level procedures. In both cohorts, patients treated with bone morphogenetic proteins were least likely to receive electrical stimulators $(p<0.05)$. Patients who received stimulation generally had higher reimbursements. Concurrent posterior lumbar fusion (PLF) (ALIF+PLF) increased the likelihood of receiving stimulators $(p<0.05)$. Patients who received electrical stimulators had similar revision rates as those who did not receive stimulation ( $p>0.05)$, except those in the multilevel ALIF+PLF cohort, wherein the patients who underwent stimulation had higher rates of revision surgery.

Conclusions: Concurrent PLF or multi-level procedures increased patients' likelihood of receiving stimulators, however, the presence of comorbidities did not. Patients who received BMA plus autograft or allograft were more likely to receive stimulation. Patients with and without bone stimulators had similar rates of revision surgery.
\end{abstract}

Keywords: Lumbar spine; Intervertebral disc degeneration; Stimulators; Grafts; Costs and cost analysis

Received Mar 12, 2018; Revised Mar 20, 2018; Accepted Apr 20, 2018

Corresponding Author: Zorica Buser

Department of Orthopaedic Surgery, Keck School of Medicine, University of Southern California, Elaine Stevely Hoffman Medical Research Center, HMR 710, 2011 Zonal Ave, Los Angeles, CA 90033, USA

Tel: +1-323-442-0206, E-mail: zbuser@usc.edu 


\section{Introduction}

Considerable evidence supports the use of electromagnetic energy to help heal long bone fractures. However, few clinical studies have examined electrical stimulators with spinal fusion, especially with anterior lumbar interbody fusion (ALIF). With the aging population, the prevalence of spinal degenerative conditions and fusion surgeries to address such pathologies has increased [1,2]. Fusion success is associated with patient outcomes; therefore, the methods to improve fusion rates may provide clinical and financial advantages [3-6].

In 1974, Dwyer and Wickham [7] published results demonstrating the benefit of direct current stimulation (DCS) for lumbar spinal fusion. Thereafter, Kane [8] conducted a randomized prospective trial wherein DCS resulted in higher fusion success rates than non-stimulated patients, even among high-risk patients. Patients are considered to be at high-risk if they were smokers, morbidly obese, diabetic, had spondylolisthesis, or were undergoing a reoperation or multi-level fusion [7-9].

Non-invasive, external alternatives to DCS include pulsed electromagnetic field stimulation (PEMFS) and capacitive coupled electrical stimulation [10]. These techniques are advantageous over DCS in terms of lower operating times, blood loss, hardware removal, and infection potential. In 1990, Mooney [11] demonstrated more favorable fusion outcomes among compliant patients with external stimulators; however, they did not stratify their results based on the anterior and posterior approaches. Moreover, other animal studies have not corroborated these results $[9,12]$. This suggests that the benefits of electrical stimulation depend on factors, such as regions of the spine or surgical approach or that improvements in surgical technique since the Mooney study have mitigated the potential benefit of the stimulators.

Recent basic science studies have proposed that external electromagnetic bone stimulators work via the upregulation of bone growth factors, including bone morphogenetic proteins (BMPs) [13]. Some grafting materials are employed for similar purposes. ALIFs can be supplemented with autograft, allograft, recombinant-human BMP, bone marrow aspirate (BMA), or a combination of these. Iliac crest bone graft (ICBG) remains the 'gold standard' of grafting materials because of its minimal immune response and optimal osteogenic, osteoconductive, and osteoinductive properties. However, it is found to have high donor site morbidity, greater neurovascular injury, and increased operation time. Allograft, BMP, and BMAs are alternatives that are less invasive alternatives; however, they lack some of bone growth qualities that make ICBG ideal $[14,15]$.

This study aimed to identify the trends in bone stimulator usage, determine the prevalence of different grafting materials used for ALIF procedures with and without stimulators, as well as compare the incidence of revision surgery between patients who did and did not receive bone stimulation. We also aimed to assess the costs associated with these procedures.

\section{Materials and Methods}

We used the PearlDiver Patient Record Database (PearlDiver Technologies Inc., Warsaw, IN, USA; http://www. pearldiverinc.com/) to follow up patients longitudinally for 1 year after their initial ALIF or ALIF+posterior lumbar fusion (PLF) surgery (ALIF+PLF=ALIF with concomitant PLF). Patient groups were divided as per the Current Procedural Terminology and the International Classification of Diseases, 9th edition insurance billing codes. We focused on patients within the database who were insured through Humana (Louisville, KY, USA) because the $\mathrm{Hu}$ mana population involved a wide age range and included nearly 20 million patients from the beginning of 2007 to the third quarter of 2015.

All the patients included in this study had lumbar disc degeneration within a year prior to their index procedure. Further, no patient had fractures or cancers of the spine.

Surgery type was classified as single- versus multi-level and ALIF-only versus ALIF+PLF. Throughout this paper, unless otherwise specified, 'ALIF' includes all patients who underwent only ALIF or ALIF+PLF procedure. To stratify patients based on the grafting material, we identified patients billed for autograft, allograft, autograft and allograft, BMA, BMP, BMA+graft, and BMP+graft on the day of their index procedure.

We determined how many patients from each group were billed for electrical stimulators within 3 months of their index ALIF procedure. Thereafter, we compared the number of patients who underwent a second surgery between those who did and did not receive stimulation. Any ALIF, PLF, PLIF, discectomy, or laminectomy performed within a year of the index ALIF was considered a revision surgery. 


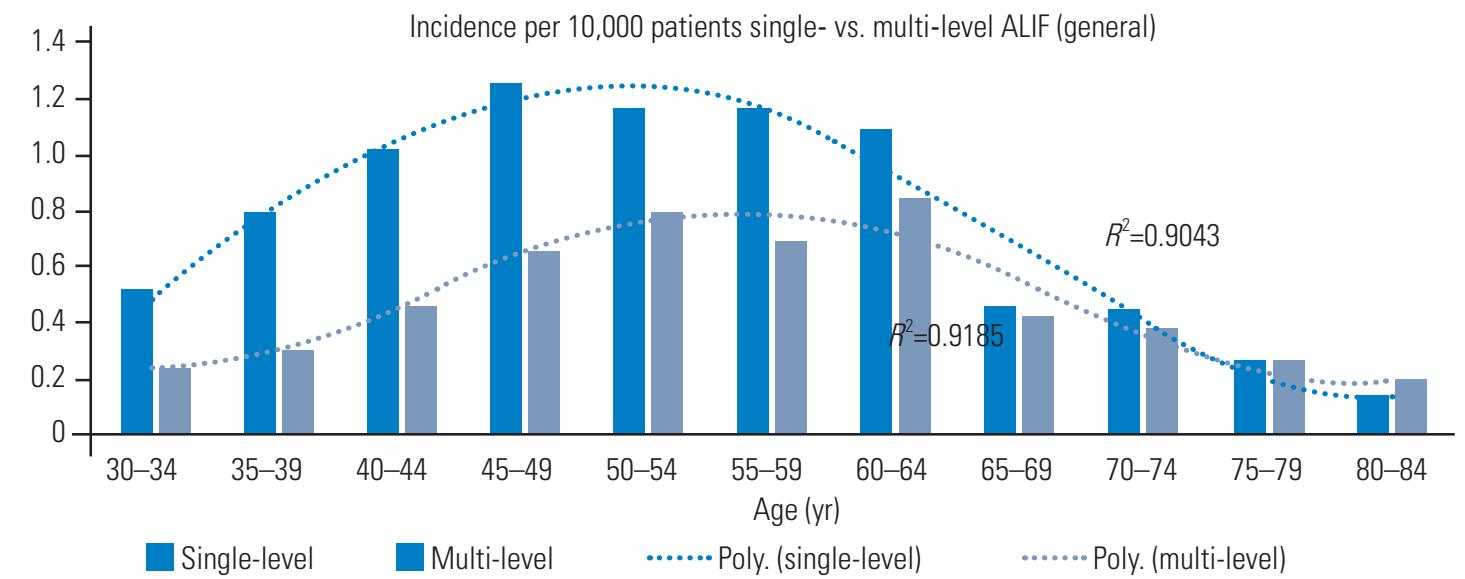

Fig. 1. Age trends among patients undergoing ALIF. Single-ALIFs are performed on a slightly younger population. ALIF, anterior lumbar interbody fusion.

Statistical analyses were performed using chi-square analysis or R software (https://www.r-project.org/) for logistic regression. Significance was set at $p<0.05$. Odds ratio (OR) data were included with a $95 \%$ confidence interval (CI) where comparisons could be made in a $2 \times 2$ contingency table.

The analyzed cost data indicate the average amount Humana reimbursed to physicians and facilities for all the costs incurred by the patient for their care over a one-day or a 1-year period from the date of surgery. Due to the data being de-identified and commercially available, no Institutional Review Board approval was needed.

\section{Results}

In the Humana subset of the PearlDiver database, between January 2007 and December 2014, 2,613 patients underwent ALIF within a year of lumbar disc degeneration diagnosis. Of those patients, 1,520 (58.2\%) were billed for single-level and 1,093 (41.8\%) were billed for multi-level ALIF. The ALIF was combined with a PLF (ALIF+PLF) in $42.4 \%$ of the single-level fusion patients and $49.6 \%$ of the multi-level fusion patients. ALIF+PLF patients underwent multi-level fusions more commonly than the ALIF-only patients ( $\mathrm{X}^{2} p<0.05$; OR, 1.590; 95\% CI, 1.36-1.86).

After adjusting for the number of patients included in the database in each age group, single-level ALIF procedures were found to be most frequent in patients aged 45-49 years, while multi-level ALIF procedures were most frequent among patients aged 60-64 years (Fig. 1). Men were slightly more likely to undergo single-level ALIF, while women were slightly more likely to undergo multi-

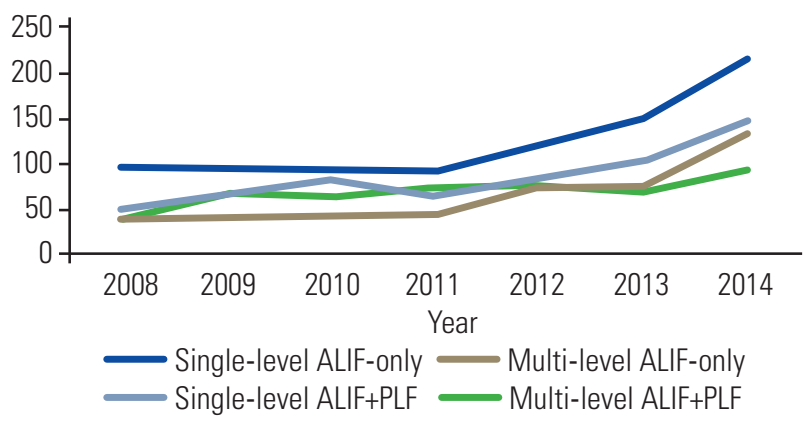

Fig. 2. An increase in the use of single- and multi-level ALIFs from 2008 to 2014, both with and without concurrent PLF procedures. ALIF, anterior lumbar interbody fusion; PLF, posterior lumbar fusion.

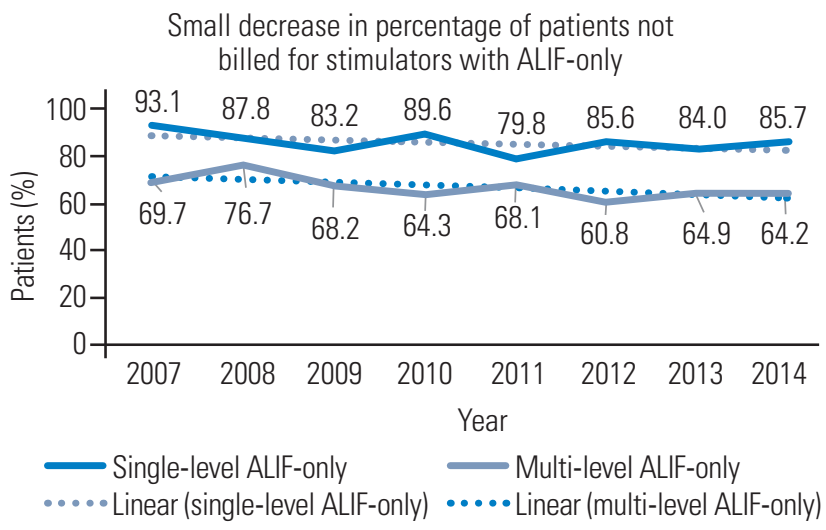

Fig. 3. Percentage of patients who did not receive stimulators following a single- or multi-level ALIF-only procedure. A minor decrease in the percentage of patients who did not receive stimulation indicates a minor increase in patients receiving stimulators following ALIF-only procedures. ALIF, anterior lumbar interbody fusion.

level ALIF ( $p<0.05$ for ALIF-only and ALIF+PLF).

The number of patients who underwent single- or multi-level ALIF procedure increased from 2008 to 2014 even after considering the rise in the population within 
Small decrease in percentage of patients not billed for stimulators with ALIF+PLF

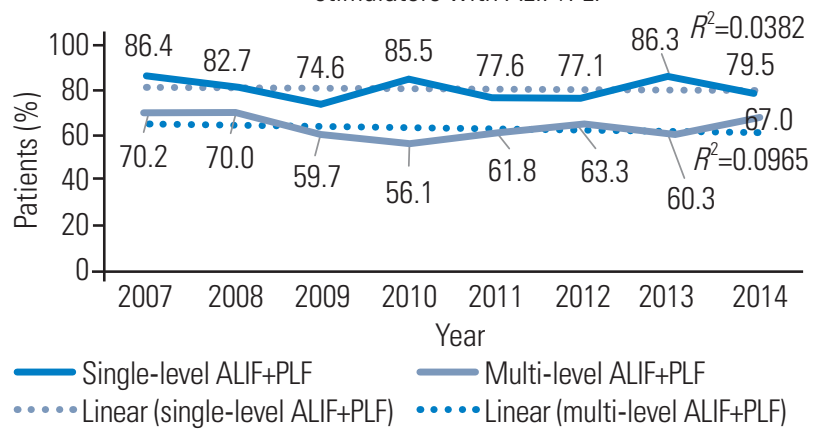

Fig. 4. Percentage of patients who did not receive stimulators following a single- or multi-level ALIF+PLF procedures. A minor decrease in the percentage of patients who did not receive stimulation indicates a minor increase in those receiving stimulators following ALIF+PLF procedures. ALIF, anterior lumbar interbody fusion; PLF, posterior lumbar fusion. the database (Fig. 2). The percentage of patients who received stimulators increased only slightly from 2008 to 2014 (Figs. 3, 4). Comorbidities, such as smoking, diabetes, and obesity that were diagnosed on the day of the index procedure did not significantly influence the likelihood of stimulator use ( $p>0.05$ for all).

Total 495 patients $(18.9 \%)$ were billed for an external stimulator within 3 months of their ALIF. No patient was billed for an internal stimulator. More patients with multilevel ALIFs (334/1,093, 30.6\%) underwent stimulation than those with single-level ALIFs $(161 / 1,520,10.6 \%)\left(\mathrm{X}^{2}\right.$ $p<0.05$; OR, 3.72; 95\% CI, 3.02-4.57). Similar trends were observed after specifying if the ALIF was performed in conjunction with a PLF $\left(X^{2} p<0.05\right.$; ALIF+PLF: 95\% OR, 1.48; 95\% CI, 1.15-1.89; ALIF-only: OR, 4.59; 95\% CI,

Table 1. Total number of patients who receive single-level, multi-level, or any ALIF, and the number and percent of those patients who were billed for an external stimulator within 3 months of that procedure

\begin{tabular}{|c|c|c|c|}
\hline Variable & Total patients & Stimulated patients (\%) & Significant difference \\
\hline ALIF-only & & & $p<0.05$ \\
\hline Single-level ALIF-only & 933 & $76(8.1)$ & \\
\hline Multi-level ALIF-only & 494 & $143(28.9)$ & \\
\hline ALIF & 1,427 & $219(15.3)$ & \\
\hline $\mathrm{ALIF+PLF}$ & & & $p<0.05$ \\
\hline Single-level ALIF+PLF & 644 & $101(15.7)$ & \\
\hline Multi-level ALIF+PLF & 542 & $174(32.1)$ & \\
\hline ALIF & 1,186 & $275(23.1)$ & \\
\hline General ALIF & & & $p<0.05$ \\
\hline Single-level ALIF & 1,520 & $161(10.6)$ & \\
\hline Multi-level ALIF & 1,093 & $334(30.6)$ & \\
\hline ALIF & 2,613 & 495 (18.9) & \\
\hline
\end{tabular}

ALIF, anterior lumbar interbody fusion; PLF, posterior lumbar fusion.

Multi-level ALIF patients were significantly more likely to be billed for stimulators for ALIF+PLF and ALIF-only patients (and ALIF in general) ( $p<0.05)$.

Table 2. Correlation of stimulator use with revision surgery

\begin{tabular}{rccccc} 
Variable & Stimulated & Revision (\%) & Non-stimulated & Revision (\%) & $p$-value \\
Single-level & & & & & \\
ALIF-only & 69 & $<11$ & 799 & $54(6.8)$ & 0.3442 \\
\hline ALIF+PLF & 91 & $<11$ & 522 & $21(4.0)$ & 0.2814 \\
Multi-level & 125 & $11(8.8)$ & 326 & $29(8.9)$ & 0.3013 \\
\hline ALIF-only & 156 & $17(10.9)$ & 342 & $13(3.8)$ & 0.0005 \\
\hline ALIF+PLF & & & & & \\
\hline
\end{tabular}

In three of four patient groups, stimulators did not appear to significantly affect the likelihood of undergoing revision surgery, and in four of four groups did not reduce the likelihood of revision surgery. Patients who received stimulators after a primary multi-level ALIF+PLF were more likely to undergo a revision surgery compared to patients who did not receive stimulators.

ALIF, anterior lumbar interbody fusion; PLF, posterior lumbar fusion. 
Table 3. Number of patients who received each type of grafting option, and the percentage of those patients who also received stimulators

\begin{tabular}{lcccc} 
& \multicolumn{2}{c}{ Single-level } & \multicolumn{2}{c}{ Multi-level } \\
\cline { 2 - 5 } Variable & Total & Stimulated patients (\%) & Total & Stimulated patients (\%) \\
Autograft & 104 & $13(12.5)$ & 100 & $40(40.0)$ \\
Allograft & 146 & $13(8.9)$ & 93 & $30(32.3)$ \\
\hline BMA & 94 & $<11(<10.6)$ & 40 & $13(32.5)$ \\
BMP & 251 & $<11(<4.0)$ & 47 & $39(25.5)$ \\
Autograft and allograft & 60 & $<11(<16.7)$ & 36 & $15(31.9)$ \\
BMA+graft & 50 & $20(40.0)$ & 61 & $14(38.9)$ \\
\hline BMP+graft & 114 & $<11(<8.8)$ & $23(37.7)$
\end{tabular}

BMA, bone marrow aspirate; BMP, bone morphogenetic protein.

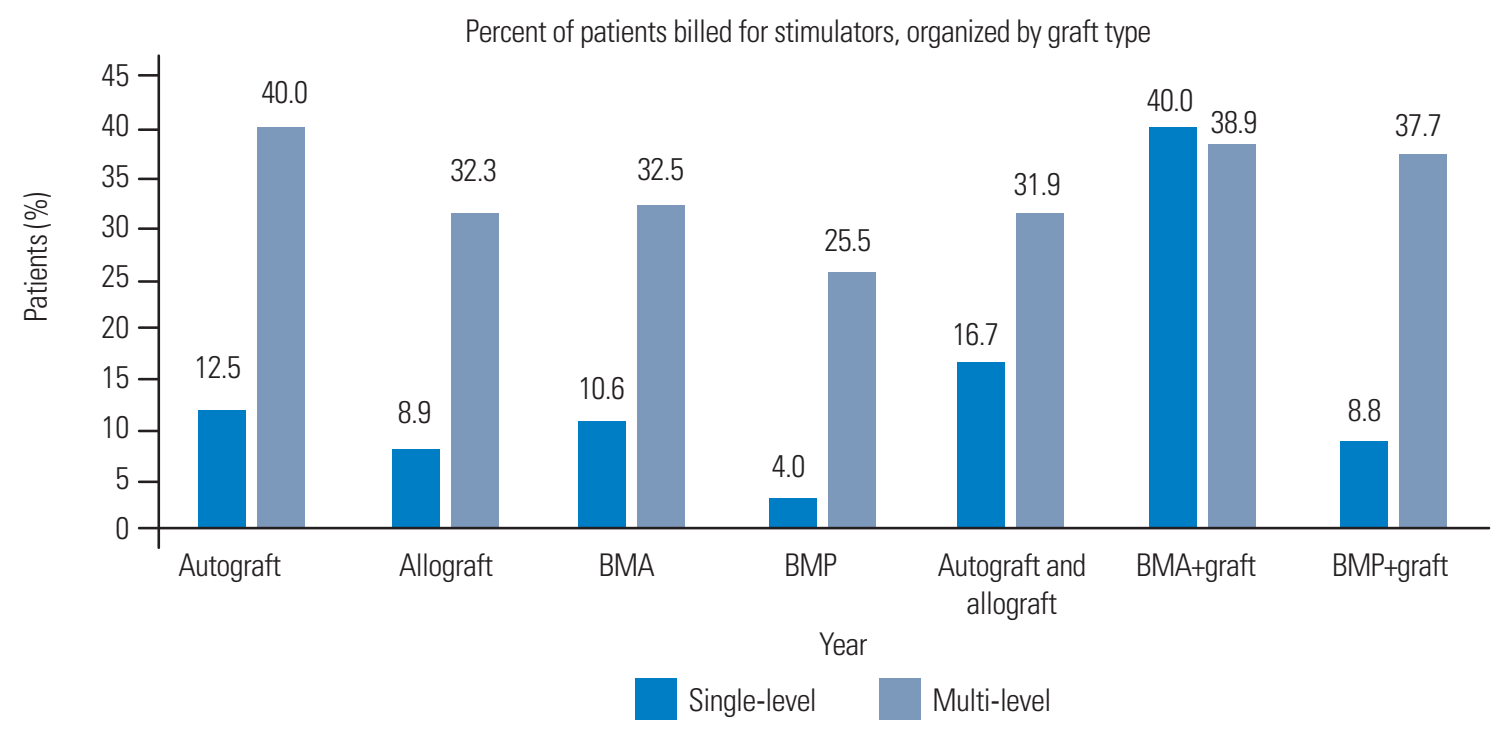

Fig. 5. Percentage of patients billed for an external stimulator after an ALIF with a specific grafting option. BMA, bone marrow aspirate; BMP, bone morphogenetic protein.

3.39-6.23) (Table 1). Single-level ALIF+PLF patients were nearly twice as likely to receive stimulators than singlelevel ALIF-only patients ( $\mathrm{X}^{2} p<0.05$; OR, 2.10; 95\% CI, 1.53-2.88). Multi-level ALIF+PLF patients were more likely to receive stimulators than multi-level ALIF-only patients; however, the result was statistically insignificant $\left(\mathrm{X}^{2} p=0.27\right.$; OR, $\left.1.161 ; 95 \% \mathrm{CI}, 0.890-1.513\right)$.

In three of the four patient groups: single-level ALIFonly, single-level ALIF+PLF, and multi-level ALIF-only, stimulators had no significant effect on the likelihood of revision surgery $\left(X^{2} p>0.05\right)$. Patients who received stimulators following a multi-level ALIF+PLF were more likely to require revision surgery $\left(\mathrm{X}^{2} p<0.05\right.$; OR, 3.10; $95 \% \mathrm{CI}$, 1.46-6.55) (Table 2).

BMP was the most common grafting option (single- level $16.5 \%$ and multi-level 14.0\%), and BMA+graft was the least common (single-level 3.3\% and multi-level 3.3\%). The number of patients who received stimulators in each of the seven different grafting subgroups is presented in Table 3. Among the single-level ALIF cohort, patients who received $\mathrm{BMA}+$ graft or allograft+autograft had a higher likelihood of receiving stimulators $(p<0.05)$. In the multilevel cohort, patients who received allograft, autograft, $\mathrm{BMA}+$ graft, or $\mathrm{BMP}+$ graft had a higher likelihood of receiving stimulators $(p<0.05)$. BMP patients consistently had a lower likelihood of receiving stimulators $(p<0.05)$ (Table 3, Fig. 5).

Patients who underwent ALIF+PLF procedures incurred higher costs than those who underwent ALIFonly procedures. Further, in patients with and without 
stimulators, the difference was more pronounced among those who received stimulators. Patients who eventually received stimulators incurred a higher cost than those who did not undergo stimulation, both on the day of the surgery and over a 1-year period. Generally, over a yearlong period (excluding the day of surgery), patients who received stimulators within 3 months of ALIF incurred a $39 \%$ and $11 \%$ higher cost than those who did not receive stimulators for single- and multi-level procedures, respectively. Notable exceptions were patients grafted with BMA and allograft or autograft. BMA+graft patients who did not receive stimulators had, on an average, $\$ 15,937$ more health-related costs after a year than similarly grafted patients who received stimulation.

\section{Discussion}

\section{General trends in stimulator usage}

The present data demonstrated variable usage of stimulators. Stimulators were used with a wide range of grafting materials and were not significantly associated with comorbidities, such as diabetes, smoking, and obesity, at the time of surgery. However, stimulators were consistently used more commonly for patients undergoing more complex procedures.

Previous studies recommended the use of electrical stimulators to aid fusion for 'high-risk' patients, including patients undergoing revision surgery or multi-level fusion $[8,9,11]$. Our data were consistent with these recommendations in that stimulators were more commonly used for patients undergoing multi-level fusion. Moreover, stimulators were used more frequently for patients undergoing ALIF+PLF rather than ALIF-only. We believe that patients undergoing ALIF+PLF represented more severe cases than those undergoing ALIF-only. Patients who received concurrent PLFs were more likely to receive stimulators for single- and multi-level procedures; however, the result was only significant for single-level procedures.

In previous studies, patients who were diabetic, obese, and smoked were also considered 'high-risk' candidates who could benefit from the use of stimulators $[8,9$, $11,16,17]$. Although two of these studies focused on internal stimulators (DCS), Mooney [11] in 1990 reported that external stimulation (PEMFS) could lower the adverse effects of smoking on fusion success. While our data did not show any significant differences in stimulator usage in patients with and without these comorbidities, it would be worthwhile to evaluate if external stimulators may benefit these patients in future studies.

\section{Trends in grafting materials used in conjunction with stimulators}

Thus far, studies that have analyzed electrical stimulators have only focused on patients treated with autograft or allograft. In 1990, Mooney [11] found similar fusion success rates for patients treated with autograft or allograft. We found that allograft, autograft, or a combination of these two were among the popular grafting options that established the use of stimulators; however, patients treated with BMA+autograft or allograft were consistently more likely to receive electrical stimulators. Single-level $\mathrm{BMA}+$ graft patients were substantially more likely to receive stimulators than patients who underwent singlelevel procedures. In contrast, multi-level BMP patients were substantially less likely to receive stimulators than patients who underwent multi-level procedures. However, the most consistent trend among the different grafting options was that patients who underwent multi-level procedures were prescribed stimulators more commonly than those who underwent single-level procedures. Other grafting options, when compared to the general population of ALIF patients, did not stand out in terms of having a clear influence on whether or not stimulators were used. While these data validate the previous discussion regarding patients who undergo multi-level procedures being more likely to receive stimulation, it also highlights the diverse population of patients who receive stimulation.

As per previous reports, patients who received BMA and autograft or allograft were prescribed stimulators more frequently $[13,18-20]$. BMA is believed to possess osteogenicity and osteoinductivity [19]. External electrical stimulators work on already differentiated cells; therefore, their use may enhance the osteogenic properties of BMA [13]. Autograft and allograft have osteoconductivity; therefore, BMA, in conjunction with autograft or allograft, may contain all the following three qualities of an ideal bone graft: osteoconductivity, osteoinductivity, and osteogenicity $[13,20]$.

\section{Costs}

From an economic perspective, patients who received 
stimulators were associated with higher costs. On an average, the total cost per patient on the day of surgery was $27 \%$ higher for single-level ALIF-only patients who would eventually receive stimulation than for those who would not. Most external stimulators are administered after the day of the surgery; moreover, stimulated patients were usually associated with higher same-day reimbursements than non-stimulated patients; therefore, we used this as a rationale to support the notion that patients who received stimulators generally represented more complex and/or challenging cases that required more time and resources.

Patients who received stimulators were also associated with greater costs over a 1-year period (excluding the day of the surgery), except for multi-level ALIF patients. The cost for multi-level ALIF patients treated with BMA+graft who received stimulators, was on an average $\$ 15,937$ lower throughout the year than that for those treated with $\mathrm{BMA}+\mathrm{graft}$ who did not receive stimulators. However, in this subgroup, the stimulated patients also had lower costs on the day of the surgery than those who did not receive stimulators, potentially indicating less complex cases to begin with. However, this is an interesting result, considering that stimulators were more commonly for patients in the BMA+graft subgroup. Future studies should seek to clarify if stimulators affect the postoperative complication rates or if their effect is minimal compared to the underlying complexity of the diagnosis.

\section{Revision surgery}

Although Mooney [11] demonstrated that external stimulators could improve the rates of fusion success in 1990, to our knowledge, no recent data are available that clearly demonstrate the benefit of stimulator use. A recent prospective study by Rogozinski et al. [21] in 2009 showed that DCS did not benefit fusion success in the elderly (age $>60$ years) lumbar fusion patients; this was contradictory to the findings of the 1988 study by Kane et al. [8] that showed that DCS improved fusion success rates [1]. Although internal stimulators work differently and are used for different procedures than external stimulators, the contrasting results of these two studies may indicate that improvements in modern surgical technique have mitigated the potential benefit of stimulators.

In large-database studies, such as the present one, the rates of revision surgery are an imperfect proxy for the estimated clinical outcomes of an index procedure. How- ever, considering their invasiveness, financial burden, and potential for further complications, revision surgeries are important clinical outcomes that physicians and patients attempt to avoid. We found that in three of the four patient groups (single-level ALIF-only, single-level ALIF+PLF, and multi-level ALIF-only), the use of stimulators following the index procedure did not significantly affect the likelihood of revision surgery. Among multilevel ALIF+PLF patients, those who underwent stimulation exhibited a significantly higher likelihood of revision surgery. We considered this as more evidence of the fact that those patients had more complicated underlying diagnoses. The null effects of stimulators on the revision rates among the other cohorts were another interesting result.

Patients may have undergone revision surgery due to several reasons beyond the scope of what we were able to determine using insurance billing codes. Our privacy contract with PearlDiver Inc. prevented us from performing a more stratified analysis of certain patient groups, such as smokers, because even with a large database, the sample size was inadequate for such analyses. However, while there might have been some patient groups for whom stimulators were beneficial, the data suggested that overall, stimulators overall did not exert a strong influence on the rates of revision surgery.

This study aimed to report, based on a large-scale perspective research, the trends that have influenced stimulator usage. It is clear that patients who undergo ALIF are diverse, and the factors that influence the use of stimulators also vary; therefore, we identified that stimulators were prescribed for more complex and challenging cases and were more frequently used with BMA plus autograft or allograft. Our data did not show any positive effect of stimulator use on the rates of revision surgery; therefore, future studies should explore specific patient populations that may benefit from stimulator use.

\section{Conclusions}

The present data indicated that physicians tend to prescribe stimulators for more complex and challenging cases, such as multi-level fusion or ALIF+PLF. Moreover, we demonstrated that stimulators were more commonly used in cases where the grafting material was BMA plus autograft or allograft. However, there was no patient group wherein the frequency of external stimulator usage 
suggested a clear standard of care.

\section{Conflict of Interest}

No potential conflict of interest relevant to this article was reported.

\section{References}

1. Deyo RA. Fusion surgery for lumbar degenerative disc disease: still more questions than answers. Spine J 2015;15:272-4.

2. Rajaee SS, Bae HW, Kanim LE, Delamarter RB. Spinal fusion in the United States: analysis of trends from 1998 to 2008. Spine (Phila Pa 1976) 2012;37:6776.

3. Carreon LY, Glassman SD, Howard J. Fusion and nonsurgical treatment for symptomatic lumbar degenerative disease: a systematic review of Oswestry Disability Index and MOS Short Form-36 outcomes. Spine J 2008;8:747-55.

4. Dimar JR 2nd, Glassman SD, Burkus JK, Pryor PW, Hardacker JW, Carreon LY. Two-year fusion and clinical outcomes in 224 patients treated with a single-level instrumented posterolateral fusion with iliac crest bone graft. Spine J 2009;9:880-5.

5. Kaiser MG, Eck JC, Groff MW, et al. Guideline update for the performance of fusion procedures for degenerative disease of the lumbar spine: part 17: bone growth stimulators as an adjunct for lumbar fusion. J Neurosurg Spine 2014;21:133-9.

6. Weinstein JN, Lurie JD, Tosteson TD, et al. Surgical compared with nonoperative treatment for lumbar degenerative spondylolisthesis. four-year results in the Spine Patient Outcomes Research Trial (SPORT) randomized and observational cohorts. J Bone Joint Surg Am 2009;91:1295-304.

7. Dwyer AF, Wickham GG. Direct current stimulation in spinal fusion. Med J Aust 1974;1:73-5.

8. Kane WJ. Direct current electrical bone growth stimulation for spinal fusion. Spine (Phila Pa 1976) 1988;13:363-5.

9. Kahanovitz N, Arnoczky SP, Nemzek J, Shores A. The effect of electromagnetic pulsing on posterior lumbar spinal fusions in dogs. Spine (Phila Pa 1976) 1994;19:705-9.

10. Oishi M, Onesti ST. Electrical bone graft stimu- lation for spinal fusion: a review. Neurosurgery 2000;47:1041-55.

11. Mooney V. A randomized double-blind prospective study of the efficacy of pulsed electromagnetic fields for interbody lumbar fusions. Spine (Phila Pa 1976) 1990;15:708-12.

12. Simons JW, Hayes MA, Cristensen KD. The effect of postoperative pulsing on lumbar fusion: an open trial study. Proceedings of the 4th Annual Meeting of the North American Spine Society; 1989 Jun 29-Jul 2; Quebec, Canada. Rosemont (IL): North American Spine Society; 1989.

13. Gan JC, Glazer PA. Electrical stimulation therapies for spinal fusions: current concepts. Eur Spine J 2006;15:1301-11.

14. Andersen T, Christensen FB, Ernst C, et al. The effect of electrical stimulation on lumbar spinal fusion in older patients: a randomized, controlled, multicenter trial: part 1: functional outcome. Spine (Phila Pa 1976) 2009;34:2241-7.

15. Johnson RG. Bone marrow concentrate with allograft equivalent to autograft in lumbar fusions. Spine (Phila Pa 1976) 2014;39:695-700.

16. Kucharzyk DW. A controlled prospective outcome study of implantable electrical stimulation with spinal instrumentation in a high-risk spinal fusion population. Spine (Phila Pa 1976) 1999;24:465-8.

17. Meril AJ. Direct current stimulation of allograft in anterior and posterior lumbar interbody fusions. Spine (Phila Pa 1976) 1994;19:2393-8.

18. Ebara S, Nakayama K. Mechanism for the action of bone morphogenetic proteins and regulation of their activity. Spine (Phila Pa 1976) 2002;27(16 Suppl 1):S10-5.

19. Flynn JM. Fracture repair and bone grafting. In: Flynn JM, editor. OKU 10: orthopaedic knowledge update. Rosemont (IL): American Academy of Orthopaedic Surgeons; 2011. p. 11-21.

20. Roberts TT, Rosenbaum AJ. Bone grafts, bone substitutes and orthobiologics: the bridge between basic science and clinical advancements in fracture healing. Organogenesis 2012;8:114-24.

21. Rogozinski A, Rogozinski C, Cloud G. Accelerating autograft maturation in instrumented posterolateral lumbar spinal fusions without donor site morbidity. Orthopedics 2009;32:809. 